\title{
Influência da função distribuição de quebra na modelagem e simulação do circuito de moagem de Carajás - Parte 1 - ajuste de modelos
}

\author{
(Influence of the distribution function on modeling and simulation of the \\ Carajás grinding circuit - Part 1 - model fit)
}

\section{Bianca Foggiatto \\ Mestranda em Engenharia Mineral Escola Politécnica da USP E-mail: bianca.foggiatto@poli.usp.br}

\section{Homero Delboni Júnior}

Professor, Doutor do Departamento de Engenharia de Minas e de Petróleo Escola Politécnica da USP E-mail:hdelboni@usp.br

\section{Resumo}

O modelo de misturador perfeito (PMM) leva em conta, tanto as características do equipamento, como as do minério. As características do minério são representadas pela função distribuição de quebra. Tal função pode ser determinada através de um método laboratorial proposto pelo JKMRC ou de funções padronizadas. O presente trabalho descreve o processo de modelagem matemática do circuito de moagem de Carajás, utilizando o software JKSimMet. Como resultado, foi possível avaliar a qualidade do ajuste do modelo em dois cenários: 1) utilizando a função distribuição de quebra-padrão do programa; 2) utilizando a função distribuição de quebra determinada para a alimentação do circuito de moagem. A partir desta análise, determinou-se, no ajuste do PMM e, em particular, na função taxa de quebra ou cinética de fragmentação, a influencia da função distribuição.

Palavras-chave: Moagem, modelagem, minério de ferro.

\section{Abstract}

The perfect mixing model (PMM) is based on parameters derived from the equipment characteristics as well as ore breakage characteristics. Ore characteristics are represented through the appearance function. This function may be determined using JKMRC laboratorial methods or by standard functions. This work describes the model fitting process of the Carajas grinding circuit, using the JKSimMet simulator. Two scenarios were used in model fitting exercises: 1) standard appearance function; and 2) appearance function based on testing carried out on samples taken at circuit feed. From this assessment, the appearance function's influence in the PMM fit and it's relation with the breakage rate were determined. The influence of the appearance function on the respective breakage rate distribution was assessed.

Keywords: Grinding, perfect mixing model, iron ore. 


\section{Introdução}

Originalmente projetado para a moagem de sinter feed, o circuito de moagem de Carajás é composto por dois moinhos de bolas em configuração fechada com ciclones, cujos overflows combinados alimentam uma etapa de deslamagem. Nesse processo, há geração significativa de finos, que são, então, descartados, acarretando em perdas de material, que, embora seja fino para ser incorporado ao produto, contém teores que atendem às especificações quanto aos contaminantes.

Além dessas perdas, esse circuito de moagem vem sendo alimentado, não apenas com sinter feed, mas, também, com ROM com qualidade para pellet feed. Esse ROM é selecionado nas frentes de lavra, fisicamente, mais próximas da usina de moagem. Atualmente o minério proveniente de N5W tem sido alimentado pela usina de moagem.

O presente trabalho descreve o processo de modelagem matemática para otimização do circuito de moagem de Carajás. Para tanto, o simulador JKSimMet foi utilizado.

O modelo matemático de cominuição empregado leva em conta, tanto as características do equipamento, como as do minério. As características do minério quanto à fragmentação são representadas pela função distribuição de quebra. Tal função pode ser determinada através de um método proposto pelo JKRMC, ou o usuário pode utilizar uma função-padrão que simulador possui.

Assim, pretende-se avaliar a qualidade do ajuste do modelo em dois cenários: 1) utilizando a função distribuição de quebra padrão do programa; 2) utilizando a função distribuição de quebra determinada para a alimentação do circuito de moagem. A influência de cada função distribuição de quebra, na respectiva cinética de fragmentação dos modelos (taxa de quebra), é, também, analisada.

\section{Modelos de processos de cominuição}

A maioria dos modelos de processos de cominuição apresenta em comum o arcabouço do Population Balance Model (PBM), ou Modelo de Balanço Populacional, que foi criado por Epstein em 1947 (apud Napier-Munn, 1996).

O PBM é um modelo de taxa de primeira ordem. Nesse modelo, assume-se que a produção de material cominuído por intervalo de tempo depende unicamente da massa da fração granulométrica considerada. Para tanto, existe uma constante, para cada fração granulométrica, que caracteriza a taxa de desaparecimento, chamada taxa de quebra (breakage rate), que é assim expressa:

$$
\text { Massa moída }=k_{i} S_{i}
$$

Onde:

$k_{i}$ é a taxa de quebra da i-ésima fração granulométrica da carga do moinho $\left(\mathrm{h}^{-1}\right)$;

$s_{i}$ é a massa da i-ésima fração granulométrica da carga do moinho $(\mathrm{t})$.

A taxa de quebra pode ser determinada a partir de ensaios de laboratório, mas, também, pode ser retrocalculada.

A equação básica do PBM tem por referência o balanço de massas para uma faixa granulométrica individual:

$f_{i}+\sum_{j=1}^{i-1} a_{i j} k_{j} s_{j}=p_{i}+k_{i} s_{i}$

Onde:

$f_{i}$ e $p_{i}$ são a vazão de sólidos da alimentação e do produto, respectivamente, correspondente a i-ésima faixa granulométrica $(\mathrm{t} / \mathrm{h})$;

$a_{i j}$ é a função distribuição de quebra.

O parâmetro função distribuição de quebra $\left(a_{i j}\right)$ representa as características do material cominuido. Trata-se da fração de uma faixa granulométrica superior (j-ésima) que, após a quebra, se reporta à faixa granulométrica considerada (i-ésima).

Uma vez determinados os parâmetros $f_{i}$ (vazão de alimentação), $p_{i}$ (vazão de produto) e $s_{i}$, e com base em uma função representativa do minério para $b_{i j}$, pode-se calcular diretamente um conjunto de valores $k_{i}$, a partir da faixa granulométrica mais grossa (Napier-Munn, 1996).

As dificuldades do PBM derivam das considerações sobre a mistura e as dificuldades em estabelecer o tempo de residência para cada fração granulométrica no interior do moinho. Com o modelo proposto por Whiten em 1976, o Modelo de Misturador Perfeito - PMM (apud Napier-Munn, 1996), essas dificuldades foram superadas ao se considerar o fenômeno como um misturador perfeito. Assim, como a carga do moinho está perfeitamente misturada, ela pode ser relacionada com o produto através de uma taxa de descarga, di, para cada faixa granulométrica.

$p_{i}=d_{i} s_{i}$

No modelo de Whiten, a função seleção é representada pela variável função quebra $r_{i}$ e a função distribuição de quebra pela variável $a_{i j}$. A equação de Whiten, que descreve o balanço para cada faixa granulométrica, é:

$f_{i}+\sum_{j=1}^{i} a_{i j} r_{i j} s_{j}=p_{i}+r_{i} s_{i}$ 
Combinadas as equações básicas do PMM, é possível normalizar os parâmetros taxa de quebra e descarga, obtendose a equação a seguir:

$$
f_{i}+\sum_{j=1}^{i}\left[\frac{a_{i j} r_{j} p_{j}}{d_{j}}\right]=p_{i}+\frac{r_{i} p_{i}}{d_{i}}
$$

O parâmetro principal do modelo, a razão $r / d$, pode ser retrocalculado diretamente por meio de amostragens e estimativas das distribuições granulométricas da alimentação e do produto do moinho, bem como através de uma função distribuição de quebra que represente as características do minério processado.

Esse parâmetro representa características do equipamento e da interação entre o equipamento e o minério.

A normalização do parâmetro é feita para corrigir variações no tempo de residência das partículas no interior do moinho. O parâmetro normalizado $\left(r / d^{*}\right)$ é representado por uma curva quadrática, definida por 3 ou 4 pontos.

\section{Materiais e métodos}

De maneira geral, na área de cominuição, os estudos voltados para a determinação de características de resistência à fragmentação de rochas e partículas pretendem avaliar a forma em que um determinado material se fragmenta.

A resistência à fragmentação é uma propriedade de complexa definição. Uma das formas mais freqüentes de avaliar tal resistência é considerar a distribuição granulométrica do produto quando um determinado mecanismo de quebra é aplicado a partículas de tamanhos definidos.

Ensaios consagrados dividem-se entre aqueles em que a fragmentação ocorre por mecanismos isolados (compressão, impacto e abrasão) e aqueles em que há combinação entre tais mecanismos. No ensaio desenvolvido pelo Julius Kruttschnitt Mineral Research Centre (JKMRC) sobre partículas individuais empregando o equipamento denominado Drop Weight Tester (DWT), a fragmentação ocorre por impacto. Esse ensaio está detalhado a seguir.

\subsection{Drop Weight Test - DWT}

Com o objetivo de analisar as características do material e aavaliar os efeitos do equipamento, foram desenvolvidos pelo JKRMC ensaios específicos sobre partículas individuais (Napier-Munn, 1996). Para a determinação da função distribuição de quebra, o ensaio-padrão é denominado de DWT.

O procedimento empregado, para determinação da função distribuição de quebra, estabelece que fragmentos sejam inicialmente classificados em faixas granulométricas predeterminadas.
Assim, são preparados três lotes para cada faixa de tamanho com número de partículas definido. A partir da massa de cada lote, as alturas de queda da carga, para cada conjunto tamanho/energia aplicada, podem ser calculadas (Chieregati, 2001).

Os fragmentos são submetidos aos impactos com energia específica entre 0,25 e 2,5 kWh/t e cada lote tem suas partículas fragmentadas individual e separadamente.

Ao final da fragmentação de cada lote, o material é reunido e peneirado. Através da distribuição granulométrica, são obtidos os parâmetros $t_{n}$, que correspondem à porcentagem passante na malha igual a $n \%$ do tamanho original do fragmento.

A relação entre energia aplicada e a fragmentação resultante para as diferentes faixas granulométricas é parametrizada da seguinte forma:

$t_{10}=A+b \cdot \ln \left(E_{c s}\right)$

Onde:

$E_{c s}$ é a energia específica aplicada ao fragmento de minério $(\mathrm{kWh} / \mathrm{t})$.

$A$ e $b$ são parâmetros característicos da resistência à quebra do minério.

Os parâmetros $A$ e $b$ são característicos da amostra ensaiada e a fragmentação é crescente conforme o aumento da energia específica aplicada à partícula, até que um limite superior seja atingido.

Assim, em função dos parâmetros $A$ e $b$, é possível calcular o $t_{10}$, para uma energia específica. A partir de valores de $t_{10}$ é possível comparar diferentes minérios e, ainda, determinar a função distribuição de quebra.

A função distribuição de quebra pode ser determinada através do gráfico desenvolvido por Narayanan (1985) apresentado na Figura 1.

Essa família de curvas representa diversos tipos de minérios e permite prever a distribuição granulométrica resultante $\left(t_{n}\right.$ 's) para um determinado nível de fragmentação conhecido $\left(t_{10}\right)$.

\subsection{Amostragens}

O circuito de moagem de Carajás foi amostrado pelos autores para obtenção de amostras que representassem seu desempenho.

A tomada de amostras, no circuito, foi realizada manualmente mediante o emprego de canecas coletoras e de técnica incremental. A coleta de incrementos foi realizada a cada 10 minutos, durante o período de 1 hora, e cada amostra foi, então, composta pelos incrementos coletados. Essa técnica foi aplicada, tanto na amostragem de fluxos de polpa, como na amostragem de fluxos de minério em correias transportadoras. 
Influência da função distribuição de quebra na modelagem e simulação do circuito de moagem de Carajás...

Foram, assim, selecionados fluxos de tomada de amostra:

- Alimentação nova e produto do moinho.

- Alimentação, underflow e overflow da classificação.

Todas as amostras, correspondentes a cada fluxo, foram analisados de forma a determinar sua distribuição granulométrica e a porcentagem de sólidos.

Os dados de vazões foram obtidos através do sistema supervisório da usina - Aspen, o qual permite obter valores médios para o período de amostragem.

\section{Resultados}

\subsection{Função distribuição de quebra}

Uma amostra da alimentação do circuito de moagem de Carajás, coletada durante a amostragem, foi submetida ao ensaio de DWT, conforme procedimento determinado por um dos autores (Delboni Jr. et al., 2001). Os ensaios foram conduzidos através de amostras com tamanho entre 22,4 e 19,0 mm. Tal faixa granulométrica foi selecionada levandose em conta o top size da alimentação dos moinhos de Carajás (50 mm).

Os resultados obtidos são apresentados na Tabela 1. Nessa mesma Tabela, são apresentados os valores de $A$ e $b$ ajustados segundo o método dos mínimos quadrados. Posteriormente, foi calculado o valor de $t_{10}$, para uma energia-padrão, nessa tabela denominado de $t_{10}{ }^{*}$.

$\mathrm{O}$ valor de $t_{10}{ }^{*}$ obtido indica que o minério alimentado pela usina no dia da amostragem apresenta uma baixa resistência ao impacto.

A função distribuição de quebra determinada é apresentada na Tabela 2.

\subsection{Balanço de massa}

Após a obtenção dos dados experimentais, eles foram submetidos a uma rotina de balanço de massas, conforme apresentado pelos autores Foggiatto e Delboni Jr. (2008).

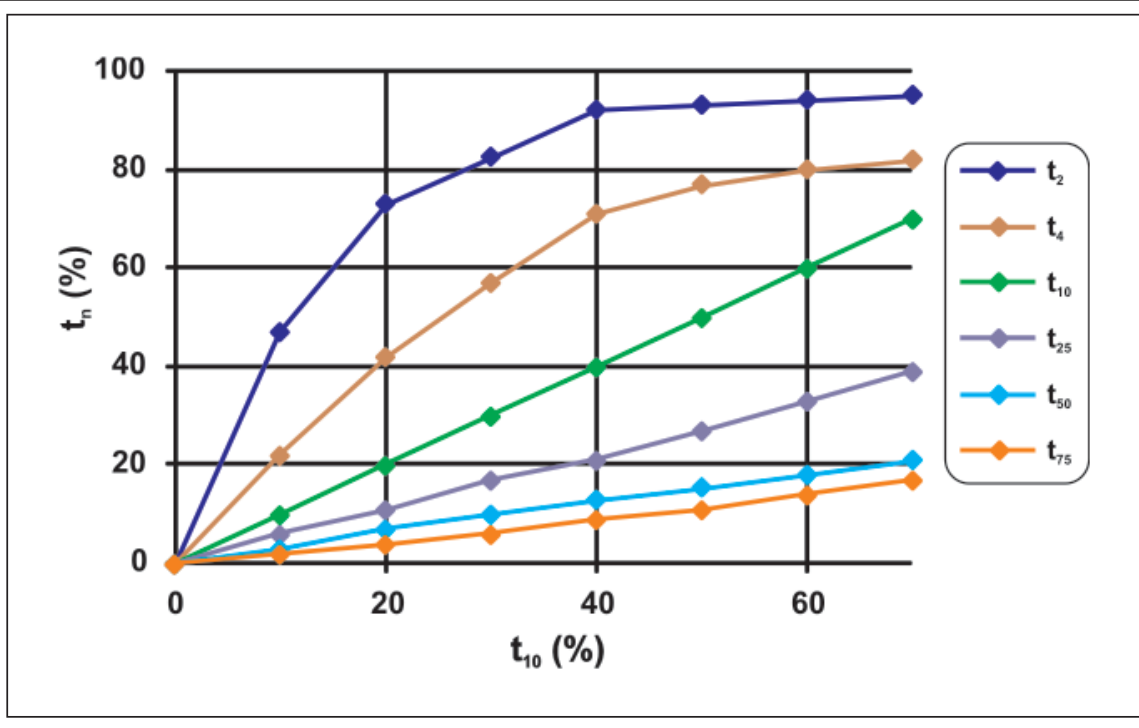

Figura 1 - Função $t_{n}$ (\% passante) vs $t_{10}$ (Adaptada de Narayanan, 1985).

Tabela 1 - Resultados obtidos nos ensaios de DWT.

\begin{tabular}{c|c|c|c|c|c}
\hline Amostra & $\boldsymbol{E}_{c \boldsymbol{s}}(\mathbf{k W h} / \mathrm{t})$ & $\boldsymbol{t}_{10}(\%)$ & $\mathbf{A}$ & $\mathbf{B}$ & $\boldsymbol{t}_{\mathbf{1 0}}{ }^{*}$ \\
\hline \multirow{2}{*}{ AL MO } & 2,53 & 46,75 & & & \\
\cline { 2 - 3 } & 1,03 & 36,85 & \multirow{2}{*}{27,0} & 10,0 & 34,6 \\
\cline { 2 - 3 } & 0,27 & 18,04 & & & \\
\hline
\end{tabular}

Como resultado do balanço de massas, dados de vazão, porcentagem de sólidos e distribuição granulométrica foram obtidos e vazões de água alimentada no circuito foram calculadas.

A Figura 2 mostra um fluxograma ilustrativo do circuito de moagem de pellet feed de Carajás, empregado no software JKSimMet.

\subsection{Modelagem}

Para o ajuste dos modelos matemáticos, foram empregados os dados obtidos no balanço de massas.

Dois ajustes foram conduzidos, empregando duas funções distribuição de quebra distintas: a primeira foi aquela determinada para o minério de Carajás (Tabela 2); e a segunda foi a função padrão do software, a qual é apresentada Tabela 2 - Função distribuição de quebra.

\begin{tabular}{c|c}
\hline & $\begin{array}{c}\text { Porcentagem retida } \\
\text { simples (\%) }\end{array}$ \\
\hline $\boldsymbol{t}_{\mathbf{1}}$ & 0 \\
\hline $\boldsymbol{t}_{\mathbf{2}}$ & 0 \\
\hline $\boldsymbol{t}_{\mathbf{4}}$ & 19 \\
\hline $\boldsymbol{t}_{\mathbf{1 0}}$ & 30 \\
\hline $\boldsymbol{t}_{\mathbf{2 5}}$ & 24 \\
\hline $\boldsymbol{t}_{\mathbf{5 0}}$ & 11 \\
\hline $\boldsymbol{t}_{\mathbf{7 5}}$ & 5 \\
\hline $\boldsymbol{f i n o s}$ & 11 \\
\hline
\end{tabular}




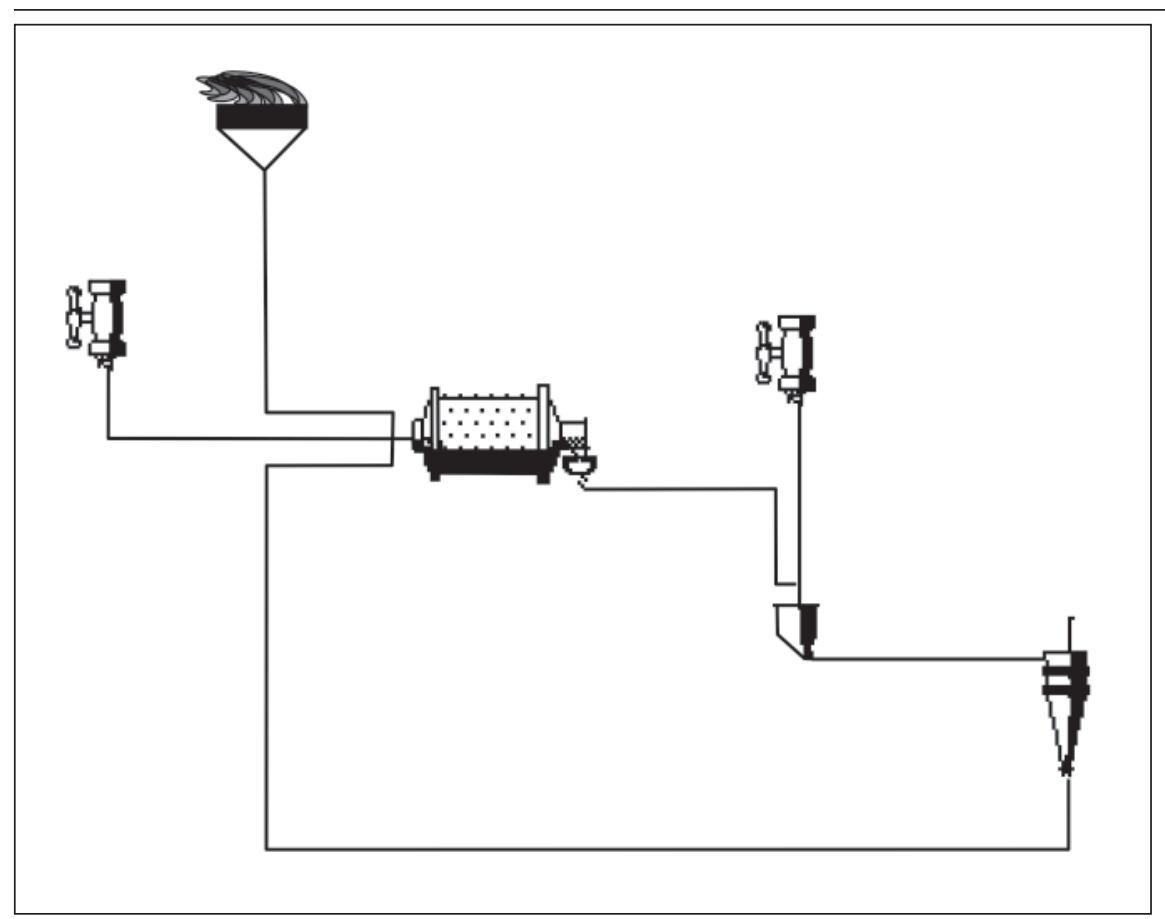

Tabela 3 - Função distribuição de quebra-padrão. Fonte: Adaptada de JKSimMet (2003).

Figura 2 - Fluxograma do circuito de moagem de Carajás.

\begin{tabular}{c|c}
\hline & $\begin{array}{c}\text { Porcentagem retida } \\
\text { simples }(\%)\end{array}$ \\
\hline $\boldsymbol{t}_{1}$ & 0 \\
\hline $\boldsymbol{t}_{2}$ & 35 \\
\hline $\boldsymbol{t}_{4}$ & 23 \\
\hline $\boldsymbol{t}_{10}$ & 18 \\
\hline $\boldsymbol{t}_{25}$ & 10 \\
\hline $\boldsymbol{t}_{50}$ & 5 \\
\hline $\boldsymbol{t}_{75}$ & 2 \\
\hline finos & 7 \\
\hline
\end{tabular}

Ao comparar as duas funções, notase que a função padrão apresenta uma concentração da geração de partículas nas frações mais grossas.

Para os ciclones, foi utilizado o modelo de curva de partição.

A somatória de erros do ajuste foi o parâmetro selecionado para determinar a qualidade de cada ajuste. Para o primeiro cenário, a somatória de erros foi de 41,45 e, para o segundo, de 39,90.

Após o ajuste, foram obtidas as taxas de quebra para os dois moinhos de Carajás, conforme o gráfico da Figura 3.

Ambas as curvas apresentam o comportamento típico da taxa de quebra: um crescimento constante até atingir um ponto de inflexão nítido, no qual a taxa de quebra máxima é atingida, crescimento que é seguido de um decréscimo para partículas mais grossas.

Nas duas curvas, o ponto de inflexão é observado para o mesmo tamanho de partículas, $3,13 \mathrm{~mm}$, porém a taxa máxima atingida foi de 340 , para o primeiro cenário, e de 435 , para o segundo cenário.

A taxa mínima de quebra atingida, para os dois cenários, são distintas. Para

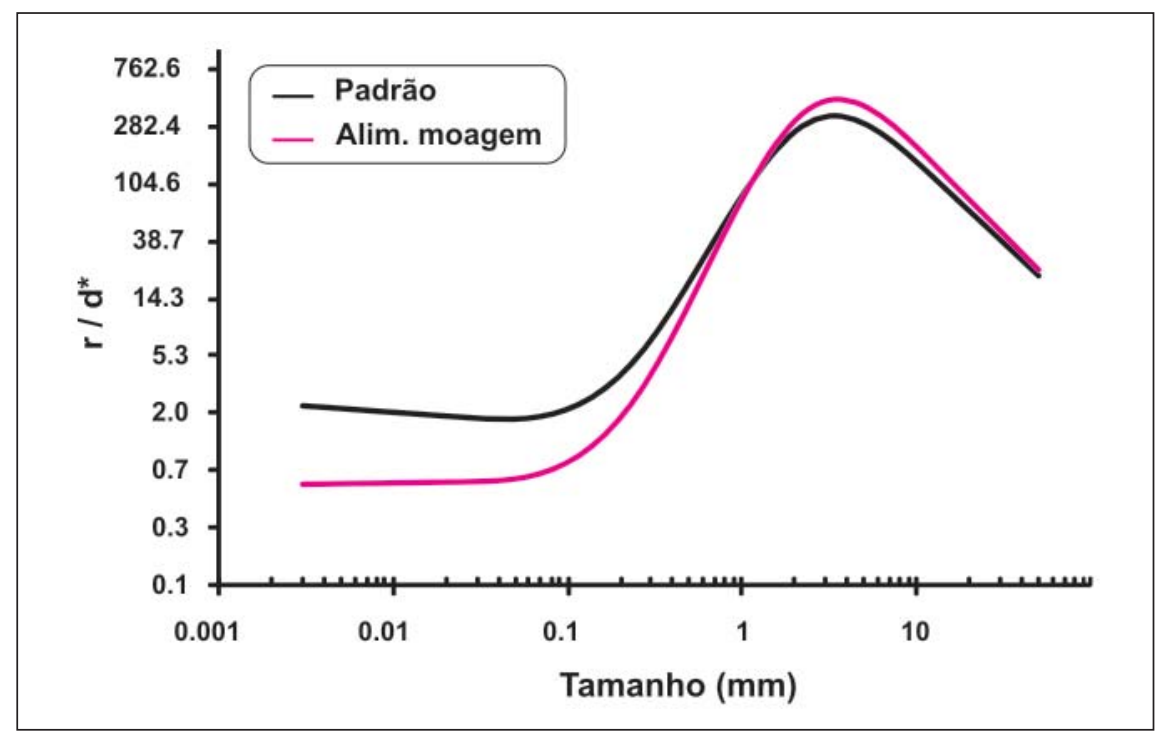

Figura 3 - Comportamento do parâmetro $r / d^{*}$ para diferentes funções distribuição de quebra.

tamanhos de partículas menores do que $0,08 \mathrm{~mm}$, a taxa de quebra é mais elevada, conforme resultados da curva-padrão, ou seja, o material mais grosso gerado em frações mais grossas é fragmentado mediante a elevação da cinética de quebra.

\section{Discussão}

Para avaliar as variações de distribuição granulométrica dos produtos obtidosm foram selecionados três parâmetros, quais sejam, a porcentagem retida em $106 \mu \mathrm{m}$ (devido à sua influência no processo de pelotização) e as porcentagens passantes em 45 e $7 \mu \mathrm{m}$. Estas últimas exercem grande influência no processo de filtragem e na área específica do pellet feed. 
Influência da função distribuição de quebra na modelagem e simulação do circuito de moagem de Carajás...

Tomando-se, como base de comparação, a função distribuição padrão do simulador, os parâmetros ajustados foram, então, avaliados. Na Tabela 4, são observados valores obtidos para o produto da moagem e para o overflow dos ciclones.

Verifica-se que, utilizando-se a função distribuição de quebra experimental, obtém-se um produto da moagem com maior desvio, em relação àquele obtido com a função-padrão. Por outro lado, o produto final do circuito (overflow dos ciclones) apresentou maiores diferenças para a função-padrão. Ainda foi possível constatar que os produtos ajustados pela função-padrão tendem a engrossar, quando comparados com os dados do balanço de massa, enquanto que os produtos gerados pela função determinada pelos autores apresentam maior quantidade de partículas finas. Como conseqüência, observa-se uma carga circulante maior para o cenário 1 do que para o cenário 2 , conforme mostra a Tabela 5.

\section{Conclusões}

A partir das análises realizadas é possível afirmar que a função distribuição de quebra exerce uma influencia direta sobre a taxa de quebra $\left(r / d^{*}\right)$ no ajuste do PMM. Ainda, nota-se que, para a função distribuição de quebra-padrão, a somatória de erros obtida foi maior.

Os produtos ajustados, para os dois cenários, apresentaram diferenças notáveis: o produto da moagem mais fino, para a função de quebra padrão, e o overflow dos ciclones mais grosso, para a função de quebra experimental.

\section{Referências bibliográficas}

FOGGIATTO, B., DELBONI JUNIOR, H., VERISSIMO, E.W. Modelling and simulating the Carajas grinding circuit. In: INTERNATIONAL MEETING ON IRONMAKING, 3. INTERNATIONAL SYMPOSIUM ON IRON ORE, 2. 2008, São Luis. Anais... São Paulo: ABM, 2008. p. 90-102. Em CD-ROM.
Tabela 4 - Parâmetros ajustados do produto da moagem e do overflow dos ciclones.

\begin{tabular}{c|c|c|c|c|c}
\hline \multirow{2}{*}{ Cenário } & \multirow{2}{*}{$\begin{array}{c}\text { Tamanho } \\
(\boldsymbol{\mu m})\end{array}$} & \multicolumn{2}{|c|}{$\begin{array}{c}\text { Passante } \\
\text { acumulado (\%) }\end{array}$} & \multicolumn{2}{c}{ Diferença (\%) } \\
\cline { 3 - 6 } & & PROD. & O/F & PROD. & O/F \\
\hline \multirow{4}{*}{$\begin{array}{c}\text { Balanço de } \\
\text { massa }\end{array}$} & 106 & 39,6 & 97,5 & - & - \\
\cline { 2 - 6 } & 45 & 19,6 & 80,8 & - & - \\
\cline { 2 - 6 } & 7 & 7,8 & 35,8 & - & - \\
\hline \multirow{3}{*}{$\begin{array}{c}\text { Função de } \\
\text { quebra padrão }\end{array}$} & 106 & 42,8 & 99,1 & $-3,2$ & $-1,6$ \\
\cline { 2 - 6 } & 7 & 19,6 & 78,9 & 0 & $-1,8$ \\
\hline \multirow{2}{*}{$\begin{array}{c}\text { Função de } \\
\text { quebra } \\
\text { experimental }\end{array}$} & 106 & 43,3 & 99,1 & $-3,7$ & $-1,6$ \\
\cline { 2 - 6 } & 4 & 22,4 & 83,4 & $+2,9$ & $+2,6$ \\
\cline { 2 - 6 } & 7 & 9,3 & 37,4 & $+1,5$ & $+1,6$ \\
\hline
\end{tabular}

Tabela 5 - Dados ajustados de porcentagem de sólidos e carga circulante.

\begin{tabular}{c|c|c|c|c}
\hline \multirow{2}{*}{ Cenário } & \multicolumn{2}{|c|}{ Porcentagem de sólidos } & \multirow{2}{*}{$\begin{array}{c}\text { Carga } \\
\text { circulante (\%) }\end{array}$} \\
\cline { 2 - 4 } & Alim. & O/F & U/F & 476 \\
\hline $\begin{array}{c}\text { Balanço de } \\
\text { massa }\end{array}$ & 58,5 & 24,4 & 82,8 & 478 \\
\hline $\begin{array}{c}\text { Função de } \\
\text { quebra padrão }\end{array}$ & 57,4 & 23,4 & 82,5 & 478 \\
\hline $\begin{array}{c}\text { Função de quebra } \\
\text { experimental }\end{array}$ & 58,1 & 25,1 & 82,7 & 442 \\
\hline
\end{tabular}

CHIEREGATI, A. C. Novo método de caracterização tecnológica para cominuição de minérios. São Paulo: Escola Politécnica, Universidade de São Paulo, 2001. 149 p. (Dissertação de Mestrado).

DELBONI JUNIOR, H., CHIEREGATI, A. C. Nova metodologia de caracterização de minérios aplicada a projetos de moinhos AG/SAG. In: ENCONTRO NACIONAL DE TRATAMENTO DE MINÉRIOS E METALURGIA EXTRATIVA, 17. Anais... Rio de Janeiro: CETEM/MCT, 2001. v. 3. p. 49-54.

JULIUS KRUTTSCHNITT TECH LTD. The JKSimMet steady state mineral processing simulator: full manual. Version 5.1. Indooroopilly, Qld, 2003. p. A69-A80.

NAPIER-MUNN, T. J. Mineral comminution circuits: their operation and optimization. Indooroopilly, Qld.: Julius Kruttschnitt Mineral Research Centre, 1996. 413 p.

NARAYANAN, S. S. Development of a laboratory single particle breakage technique and its application to ball mill modelling and scale-up. Queensland: Julius Kruttschnitt Mineral Research Centre, University of Queensland, 1985. 209 p. (Tese de Doutorado).

Artigo recebido em 26/12/2008 e aprovado em 27/10/2009. 